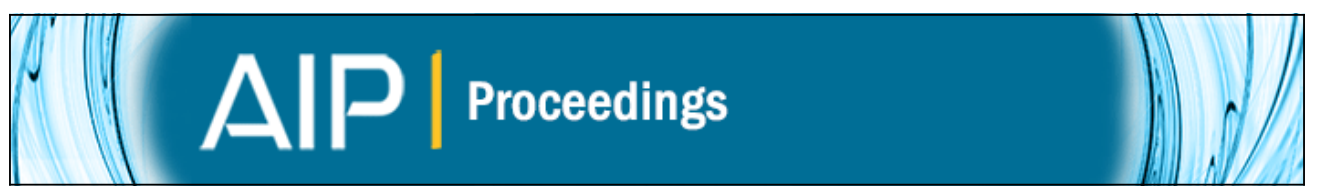

\title{
Magnetized Plasma-filled Waveguide: A New High-Gradient Accelerating Structure
}

Yoav Avitzour and Gennady Shvets

Citation: AIP Conference Proceedings 1086, 508 (2009); doi: 10.1063/1.3080959

View online: http://dx.doi.org/10.1063/1.3080959

View Table of Contents:

http://scitation.aip.org/content/aip/proceeding/aipcp/1086?ver=pdfcov

Published by the AIP Publishing

\section{Articles you may be interested in}

Emittance and Current of Electrons Trapped in a Plasma Wakefield Accelerator

AIP Conf. Proc. 1086, 591 (2009); 10.1063/1.3080974

Measurement of the Decelerating Wake in a Plasma Wakefield Accelerator AIP Conf. Proc. 1086, 569 (2009); 10.1063/1.3080969

Electron acceleration in a warm magnetized plasma-filled cylindrical waveguide J. Appl. Phys. 104, 073303 (2008); 10.1063/1.2988899

Electron dynamics and acceleration study in a magnetized plasma-filled cylindrical waveguide

J. Appl. Phys. 103, 023302 (2008); 10.1063/1.2831223

Guided modes and loss in a plasma-filled Bragg waveguide

J. Appl. Phys. 102, 063304 (2007); 10.1063/1.2776374 


\title{
Magnetized Plasma-filled Waveguide: A New High-Gradient Accelerating Structure
}

\author{
Yoav Avitzour and Gennady Shvets \\ Department of Physics and Institute for Fusion Studies, The University of Texas at Austin, One \\ University Station C1500, Austin, Texas 78712
}

\begin{abstract}
Electromagnetic waves confined between the metal plates of a plasma-filled waveguide are investigated. It is demonstrated that when the plasma is magnetized along the metallic plates, there exists a luminous accelerating wave propagating with a very slow group velocity. It is shown that the magnetized plasma "isolates" the metal wall from the transverse electric field, thereby reducing potential breakdown problems. Applications of the metallic plasma-filled waveguide to particle accelerations and microwave pulse manipulation are described.
\end{abstract}

Keywords: millimeter waves, high-gradient acceleration

PACS: $52.35 . \mathrm{Mw}, 52.38 .-\mathrm{r}, 52.38 . \mathrm{Hb}$

\section{INTRODUCTION AND MOTIVATION}

The quest to higher accelerating gradients inevitably points to higher frequencies of the accelerating field. This is because, according to the dark current trapping criterion, the accelerating gradient $W_{z}<m c \omega / e$, where $\omega$ is the angular frequency, and $e$ and $m$ are the electron charge and mass, respectively. In a conventional accelerating structure higher frequencies also imply smaller feature size which are hard to fabricate, and can be easily damaged by single-pulse Ohmic heating and the resulting cyclic stress [1]. At the other end of the frequency spectrum are the laser-driven plasma accelerators. Laser wakefield and plasma beatwave accelerators require short-pulse high power laser pulses because plasma wave is driven via the nonlinear ponderomotive force. Typical plasma densities are of order $10^{17}-10^{19} \mathrm{~cm}^{-3}$, and plasma wavelength $10 \mu \mathrm{m}<\lambda_{p}<100 \mu \mathrm{m}$. Low laser efficiency and repetition rate are some of the technical challenges on the path to developing a practical laser-plasma accelerator.

In this paper we look at the alternative regime of high-gradient acceleration that combines an electromagnetic structure (metallic waveguide) and the plasma medium. Intermediate frequencies (hundreds of $\mathrm{GHz}$ ) and plasma densities (of order $10^{14}$ $10^{15} \mathrm{~cm}^{-3}$ ) can be utilized, and high power microwaves can be directly converted into plasma waves. The idea of using magnetized plasmas for slowing down electromagnetic waves, converting their polarization into mostly longitudinal one, and then employing the wave for particle acceleration has been discussed [2] before in the context of the Undulator Induced Transparency (UIT) [3, 4, 5]. UIT utilizes a transverse magnetic field $\boldsymbol{B}_{u}$ to couple the transverse and longitudinal waves in a uniformly magnetized plasma $\left(\boldsymbol{B}_{0}=B_{0} \boldsymbol{e}_{z}\right)$ in order to slow them down. The slowing-down occurs because the resulting hybrid wave is primarily longitudinal and, therefore, suitable for particle acceleration. The transverse magnetic field can either be helical as envisioned in the original $[3,4$, 


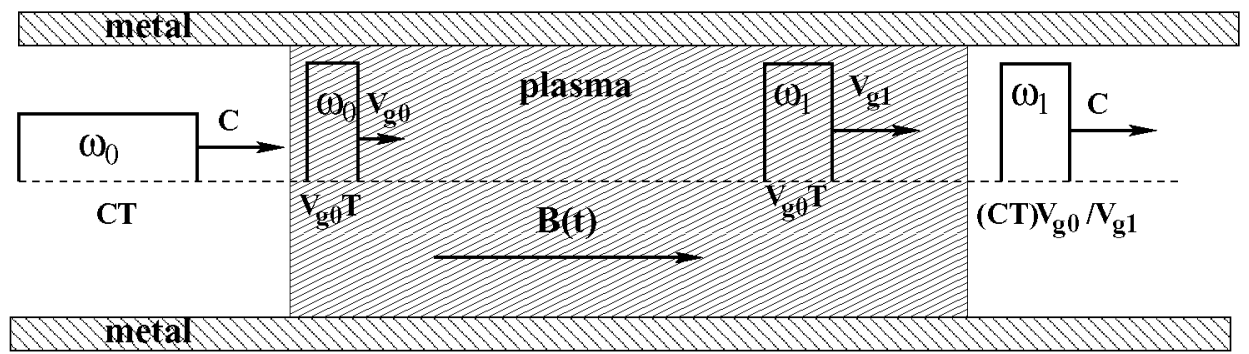

FIGURE 1. Schematic of the magnetized plasma-filled waveguide (MPW) that can be used for either particle acceleration, or for compressing microwave pulses. The latter application is illustrated here. Radiation pulse with initial frequency $\omega_{0}$ and duration $T$ slows down in the plasma to a group velocity $v_{g 0} \ll c$. Uniform in space adiabatic in time (USAT) variation of the magnetic field changes the radiation frequency to $\omega_{1}$ and increases the group velocity to $v_{g 1} \gg v_{g 0}$ of plasma-trapped radiation. The emerging pulse is compressed to $T_{1}=T v_{g 0} / v_{g 1}$.

5] UIT papers, or linear as was suggested for the Inverse Cherenkov Accelerator in Magnetized Plasma (ICAMP) [2]. Simple one-dimensional theories of both magnetic configurations were developed in Ref. [2], and the main drawback of using the transverse magnetic field was identified: synchrotron radiation by the accelerated particles.

In this paper we demonstrate that a purely longitudinal magnetic field $\left(\boldsymbol{B}_{0}=B_{0} \boldsymbol{e}_{z}\right.$ ) aligned with the direction of the particles' acceleration is sufficient for slowing down electromagnetic waves when the plasma is confined by the metallic walls of a waveguide as shown in Fig. 1. We demonstrate that the group velocity of the resulting electromagnetic wave can simultaneously have a luminous phase velocity $\omega / k_{z}=c$ and a slow group velocity $v_{g} / c \ll 1$ when the operating frequency is chosen to be $\omega \approx \omega_{p}$, where $\omega_{p}=\left(4 \pi e^{2} n_{0} / m\right)^{1 / 2}$ is the electron plasma frequency. This configuration, referred to as the Magnetized Plasma-filled Waveguide (MPW) has several distinct advantages over the ones requiring a transverse magnetic field. First, particles accelerated along the $z$-direction do not emit any synchrotron radiation. Second, plasma is transversely confined by the waveguide walls. Third, MPW can be utilized for dynamic manipulation of microwave pulses [6] as illustrated in Fig. 1: increasing the magnitude of the magnetic field $B_{0}$ has the effect of increasing the group velocity of the radiation, thereby shortening the duration of the microwave pulse released from the plasma. Therefore, an MPW can serve as either an accelerator or a microwave power compressor. A metal structure has a when compared with the purely plasma-based scheme: finite transverse electric field at the metal wall may result in the breakdown of the structure. Fortunately, it is shown (see Fig. 2(b)) that the transverse component of the electric field on the wall is very small: almost two orders of magnitude smaller than the peak accelerating field. In other words, magnetized plasma "insulates" the metal wall. 


\section{EIGENMODES OF THE MAGNETIZED PLASMA-FILLED WAVEGUIDE}

In this Section we develop the theoretical framework for determining the modes of a plasma-filled metallic waveguide. For simplicity, assume (see Fig. 1) that the waveguide and plasma are infinite in the $y$-direction and that the metallic plates are placed at $x=0$ and $x=L$. Plasma is assumed to be uniformly magnetized in the $z$-direction, which is also the propagation direction of both the microwaves and the accelerated particles. Qualitatively, having an electromagnetic wave confined between two metal plates is equivalent to propagating it an angle $\theta_{m}=\tan ^{-1}\left(k_{m} / k_{z}\right)$ with respect to $\boldsymbol{B}_{0}$, where $k_{m}=m \pi / L$ is the transverse wavenumber, and $m$ is an integer number labelling the specific propagation mode. This simplistic model is indeed accurate for the waveguides filled with unmagnetized plasma. For the magnetized plasma, however, the situation is more complicated: the spatial spectrum of a given mode contains a spectrum of transverse wavenumbers $k_{m}$.

Assuming $\boldsymbol{E}(\boldsymbol{x}, t)=\tilde{\boldsymbol{E}}(x) \exp \left(i k_{z} z-i \omega t\right)$, a generalized eigenvalue equation for $\tilde{\boldsymbol{E}}$ is obtained:

$$
\left\{\left[\begin{array}{ccc}
k_{z}^{2} & 0 & i k_{z} \partial_{x} \\
0 & -\partial_{x}^{2}+k_{z}^{2} & 0 \\
i k_{z} \partial_{x} & 0 & -\partial_{x}^{2}
\end{array}\right]-k_{0}^{2} \boldsymbol{\varepsilon}\right\} \cdot \tilde{\boldsymbol{E}}=0
$$

where $k_{0} \equiv \omega / c$, and the dielectric permittivity tensor $\boldsymbol{\varepsilon}$ of the cold magnetized plasma is given by

$$
\boldsymbol{\varepsilon}=\left[\begin{array}{ccc}
\varepsilon_{1} & i \varepsilon_{2} & 0 \\
-i \varepsilon_{2} & \varepsilon_{1} & 0 \\
0 & 0 & \varepsilon_{3}
\end{array}\right]
$$

where $\varepsilon_{1}=1-\omega_{p}^{2} /\left(\omega^{2}-\Omega_{0}^{2}\right), \varepsilon_{2}=\omega_{p}^{2} \Omega_{0} / \omega\left(\omega^{2}-\Omega_{0}^{2}\right)$ and $\varepsilon_{3}=1-\omega_{p}^{2} / \omega^{2}$. Here $\Omega_{0}=e B_{0} / m c$ is the electron cyclotron frequency.

Eliminating $\tilde{E}_{x}$ results in the two coupled ODEs:

$$
\left[\partial_{x}^{2}+A\right] \tilde{E}_{y}=B \partial_{x} \tilde{E}_{z} \text { and }\left[\partial_{x}^{2}+C\right] \tilde{E}_{z}=D \partial_{x} \tilde{E}_{y}
$$

where $A=\gamma-\varepsilon_{2}^{2} k_{0}^{4} / \gamma, B=-k_{z} \varepsilon_{2} k_{0}^{2} / \gamma, C=\gamma \varepsilon_{3} / \varepsilon_{1}, D=k_{z} \varepsilon_{2} / \varepsilon_{1}$, and $\gamma=\varepsilon_{1} k_{0}^{2}-k_{z}^{2}$. After expanding $\tilde{E}_{y}$ and $\tilde{E}_{z}$ as

$$
\left[\begin{array}{l}
E_{y} \\
E_{z}
\end{array}\right]=\sum_{n=1}^{\infty}\left[\begin{array}{l}
a_{n} \\
b_{n}
\end{array}\right] \sin k_{n} x
$$

and inserting Eq. (4) into Eq. (3), the following implicit dispersion relation is obtained:

$$
\begin{aligned}
& a_{m}\left(A-k_{m}^{2}\right)=\frac{4 B}{L} \sum_{n+m \text { odd }} \frac{k_{m} k_{n}}{k_{m}^{2}-k_{n}^{2}} b_{n} \\
& b_{m}\left(C-k_{m}^{2}\right)=\frac{4 D}{L} \sum_{n+m \text { odd }} \frac{k_{m} k_{n}}{k_{m}^{2}-k_{n}^{2}} a_{n} .
\end{aligned}
$$



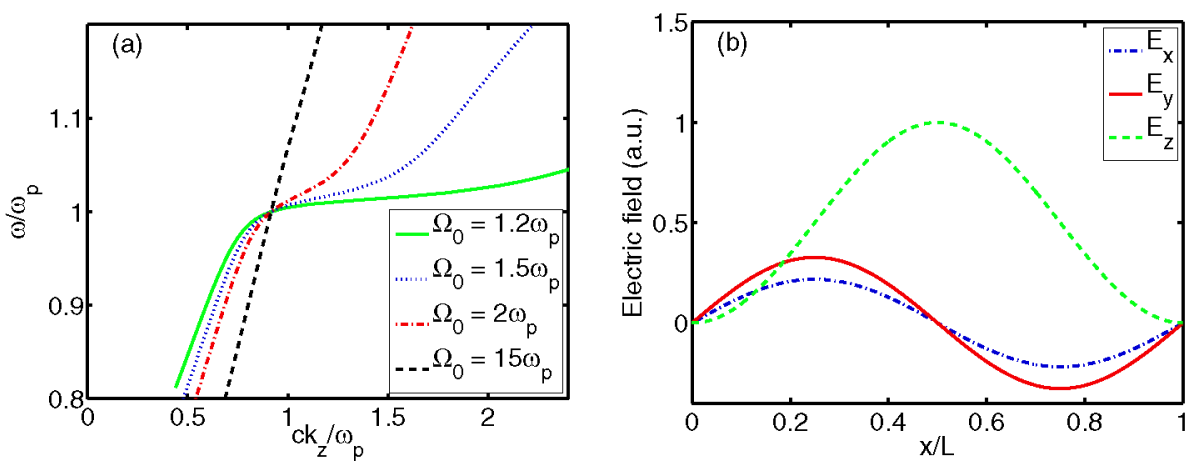

FIGURE 2. (a) Family of dispersion curves and (b) electric field profiles (for $\omega=\omega_{p}$ and $\Omega_{0}=1.5 \omega_{p}$ ) of one of the modes of an overmoded plasma-filled waveguide of width $L=5 \pi c / \omega_{p}$.

Even though Eqs. (5) must be solved numerically by truncating the number of spatial modes to $1 \leq m, n \leq N$, simple inspection reveals several features of the solution. First, there are no single-mode solutions. Second, if, for example, the $E_{y}$ component is dominated by the $k_{1}$ mode, then the $E_{z}$ component is dominated by the $k_{2}$ mode, and vice versa, i.e., the dominant mode is different for the $E_{z}$ and $E_{y}$ components of the field. Truncated Eqs. (5) were numerically solved as a generalized nonlinear eigenvalue problem of the $4^{t h}$ order for $k_{z}$. It is found from the numerical solution that the $E_{y}$ and $E_{z}$ field components of the slowest mode propagating in the MPW mostly correspond to the $m=2$ and $m=1$ transverse spatial modes, respectively. An overmoded waveguide of the width $L=5 \pi c / \omega_{p}$ was chosen. Mode profiles of $E_{y}$ and $E_{z}$ are plotted in Fig. 2(b) for $\omega=\omega_{p}$ and $\Omega_{0}=1.5 \omega_{p}$. The dispersion curves $\omega$ vs $k_{z}$ for the waves propagating in an MPW are plotted in Fig. 2(a) for different values of $\Omega_{0}$.

Several features of the accelerating mode are apparent from Fig. 2(b). First, the accelerating component of the electric field $E_{z}$ is considerably higher (by a factor 3 in this example) than its transverse components $E_{x}$ and $E_{y}$. Second, $E_{x}$ at the wall is almost two orders of magnitude smaller than the peak accelerating field. This is important for avoiding potential breakdown problems. The physical reason for the smallness of $E_{x}$ at the wall is that the magnetized plasma forces the waves to be almost circularly polarized. Because $E_{y}$ must vanish at the perfectly-conducting wall, the magnitude of $E_{x}$ is similarly reduced. Third, even though $E_{x}$ is smaller than $E_{z}$, in is still quite sizeable and can, in principle, be used for particle focusing. Because fairly large accelerating gradients (hundreds of $\mathrm{MeV} / \mathrm{m}$ ) are envisioned, the focusing strength of the accelerating mode can be higher than that provided by conventional magnets.

The dispersion curves plotted in Fig. 2(a) also reveal several attractive features of the accelerating mode. First, the accelerating mode can simultaneously have a slow group velocity and a luminous phase velocity. It is found that $v_{g} / c \approx 1 / 60$ for $\Omega_{0}=1.2 \omega$ and $v_{g} / c \approx 1 / 10$ for $\Omega_{0}=1.5 \omega$. Low group velocity corresponds to considerable compression of the microwave energy and conversion of the incident transverse electric field into the longitudinal one. Second, it is evident from inspecting Fig. 2(a) that, for $\Omega_{0}=1.2 \omega$, low group velocity persists even for relatively low phase velocities $\omega / c k_{z} \approx$ 
0.4. That implies that the accelerating modes of the MPW can be used for accelerating high energy ions. Finally, we observe that, regardless of the magnitude of the magnetic field, all dispersion curves in Fig. 2(a) intersect at the common point $\omega=\omega_{p}$ and $c k_{z}=\omega_{p}$. As demonstrated below, this fact can be used for compressing microwave pulses in the MPW by increasing the magnitude of the magnetic field uniformly in space and adiabatically in time. Microwave pulses can be compressed without frequency shift if $\omega=\omega_{p}$, and with the frequency upshifting if $\omega>\omega_{p}$.

\section{DYNAMIC MANIPULATION OF MICROWAVE PULSES USING MAGNETIZED PLASMA-FILLED WAVEGUIDES}

Another conclusion that can be drawn from Fig. 2(a) is that the group velocity of the electromagnetic wave inside the MPW can changed by slowly changing the magnitude of the magnetic field. Assume that the electromagnetic wave has the frequency $\omega_{0}$ and the propagation wavenumber $k_{0}=k_{0} e_{z}$. Then a spatially uniform change of the magnetic field cannot change the propagation wavenumber $k_{0}$. On the other hand, the frequency can, generally, change in response to the magnetic field's changing from $B_{0}$ to $B_{1}$. If the change occurs on a time scale much slower than the radiation frequency, then no mode conversion can occur. Therefore, the dispersion curve of the mode will slowly evolve from $\omega(k)_{B_{0}}$ to $\omega(k)_{B_{1}}$. An analogous "frequency adjustment" occurs when the length of an oscillating pendulum is slowly (adiabatically) changed.

The family of the dispersion curves $\omega(k)_{B}$ for various values $B$ of the axial magnetic field is shown in Fig. 2(a). Subject to the conservation of the spatial wavenumber $k_{0}$, the mode's frequency then evolves from $\omega\left(k_{0}\right)_{B_{0}} \equiv \omega_{0}$ to $\omega\left(k_{0}\right)_{B_{1}} \equiv \omega_{1}$. Because all dispersion curves intersect at $\omega=\omega_{p}$, one can conclude that the variation of the magnetic field does not affect the frequency of the wave when $\omega_{0}=\omega_{p}$. Pulse duration, however, can be significantly compressed when $B_{1}>B_{0}$. To understand the phenomenon of pulse compression, imagine a finite-length pulse with the spatial spectral bandwidth $\Delta k: k_{0}-\Delta k / 2<k<k_{0}+\Delta k / 2$. The corresponding temporal bandwidth is $(\Delta \omega)_{0}=$ $\Delta k(d \omega / d k) \equiv \Delta k v_{g 0}$, where $v_{g 0}$ is the initial group velocity. After changing the magnetic field uniformly in space, the spatial bandwidth $\Delta k$ remains the same while the temporal bandwidth becomes $(\Delta \omega)_{1}=v_{g 1} \Delta k \gg(\Delta \omega)_{0}$ if $v_{g 1} \gg v_{g 0}$. The resulting bandwidth increase translates into the proportional shortening of the temporal duration of the pulse.

To demonstrate the USAT compression of microwave pulses in an MPW configuration, the time-dependent Maxwell's equations with linearized plasma response have been solved numerically for $\Omega_{0}$ increasing adiabatically from $\Omega_{0}=1.5 \omega_{p}$ to $\Omega_{0}=15 \Omega_{p}$. We assumed $\partial_{y}=0, \partial_{z}=i k_{z}$ and solved the one-dimensional time-dependent PDE using the standard leapfrog method. The system is driven initially by an external current pulse at a fixed frequency $\omega_{0}$, and the appropriate $k_{z}$ is determined by the numerical solution of Eqs. (5). Simulation results presented in Fig.3 demonstrate that in the MPW configuration (i) group velocity can be increased by an order of magnitude by increasing the magnetic field in the same proportion, (ii) that the spatial field profile remains essentially unchanged in the course of USAT, and (iii) the frequency of the compressed pulse remains the same for $\omega_{0}=\omega_{p}$. As a practical example, consider the compression of an

$\omega / 2 \pi=3 \mathrm{GHz}$ pulse in a magnetized plasma of density $n_{0}=10^{11} \mathrm{~cm}^{-3}$ placed inside 

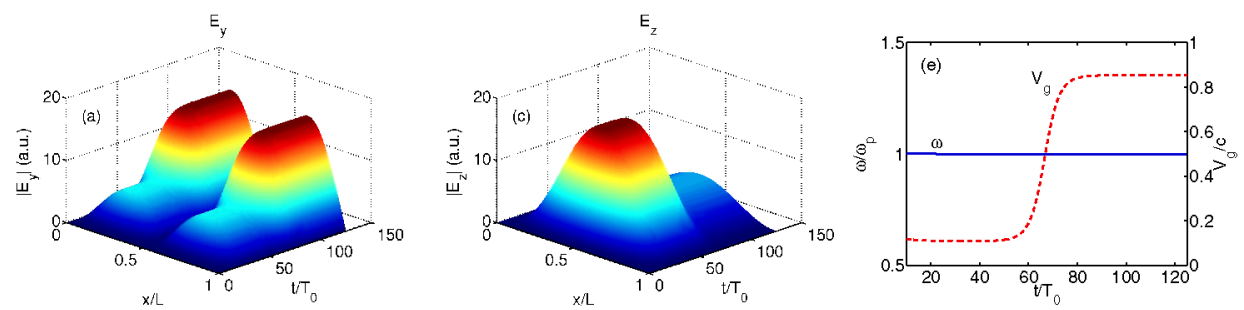

FIGURE 3. Time evolution of the slow mode of the MPW $\left(L=5 \pi c / \omega_{p}\right)$ with $\omega_{0}=\omega_{p}$ during the variation of the magnetic field: $1.5<\Omega / \omega_{p}<15$. Left: transverse electric field, middle: longitudinal electric field, right: group velocity and frequency.

a waveguide of width $L=25 \mathrm{~cm}$ using the USAT approach. By adiabatically increasing the magnetic field from $B_{0}=0.15 \mathrm{~T}$ to $1.5 \mathrm{~T}$, pulse compression by an order of magnitude can be accomplished.

\section{CONCLUSIONS}

In conclusion, we have demonstrated that a Magnetized Plasma-filled Waveguide (MPW) supports slow accelerating plasma waves. The magnetized plasma effectively "insulates" the waveguide wall from the transverse electric field that could otherwise contribute to the structure's breakdown. By slowly increasing the amplitude of the magnetic field inside the MPW, trnasmitted microwave pulses can be compressed in time. Future work will address the coupling efficiency of microwaves into the MPW, as well as the nonlinear effects that occur when the electric field approaches the cold plasma wave-breaking limit.

\section{ACKNOWLEDGMENTS}

This work is supported by the U.S. Department of Energy grants No. DE-FG0204ER54763 and DE-FG02-04ER41321.

\section{REFERENCES}

1. Whittum, D. H., "Ultimate Gradient in Solid State Accelerators," in Advanced Accelerator Concepts Workshop: Eighth Workshop, edited by W. Lawson, C. Bellamy, and D. F. Brosius, American Institute of Physics, 1999, vol. 472, p. 72.

2. Shvets, G., WUurtele, J. S., and Hur, M.-S., "Applications of Magnetized Plasma to Particle Acceleration," in Advanced Accelerator Concepts: Tength Workshop, edited by C. E. Clayton and P. Muggli, American Institute of Physics, 2002, vol. 647, p. 681.

3. Shvets, G., and Wurtele, J. S., Phys. Rev. Lett., 89, 115003 (2002).

4. Hur, M., Wurtele, J., and Shvets, G., Physics of Plasmas, 10, 3004-3011 (2003).

5. Tushentsov, M., Shvets, G., Kryachko, A., and Tokman, M., IEEE Trans. Plasma Science, 33, 23-31 $(2005)$.

6. Avitzour, Y., and Shvets, G., Phys. Rev. Lett., 100, 065006 (2008). 